\title{
PROPERTIES OF THE SOLAR FILIGREE STRUCTURE
}

R. B. DUNN, J. B. ZIRKER*, and J. M. BECKERS

Sacramento Peak Observatory, AFCRL, Sunspot, N.M. 88349, U.S.A.

\begin{abstract}
A number of observers have noted the presence of bright structures near the cores of the chromospheric rosettes when observed in the far wings of the $\mathrm{H} \alpha$ line (eg $\mathrm{H} \alpha \pm 7 / 8 \AA$ ). Dunn and Zirker observed these bright structures with the highest possible resolution using the Sacramento Peak vacuum solar telescope. They find that these bright regions exhibit a very intricate fine structure which can be followed out much further into the $\mathrm{H} \alpha$ line wing (eg $\mathrm{H} \alpha+2 \AA)$ and even into the continuum. They called this fine structure 'solar filigree', the name referring mainly to the collective appearance of the fine structure elements. The elements themselves appear as dot-like structures and frequently also as small wiggly structures called 'crinkles'. The propr rrties of the filigree structure are summarized as follows:

(i) Size: Measured diameter of the crinkles and dots equals $0.25,0.40$ and $0.60^{\prime \prime}$ at $\mathrm{H} \alpha+2 \AA, \mathrm{H} \alpha \pm 7 / 8 \AA$ and $\mathrm{H} \alpha \pm 5 / 8 \AA$ respectively. The telescope resolution equals 0.22 " so that at $\mathrm{H} \alpha+2 \AA$ the structure is extremely small. The drawings in Figure 1 show typical sizes of the crinkles and network patterns in the filigree.
\end{abstract}
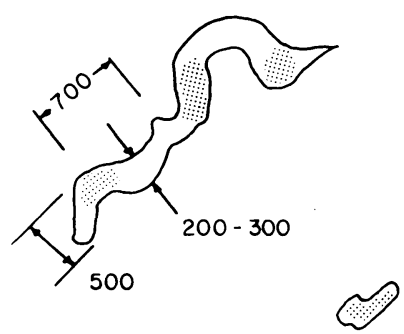

Fig. 1. Typical size of filigree patterns.

(ii) Contrast : Filigree is enhanced in the blue wing of the $\mathrm{H} \alpha$ line. Measured contrast, uncorrected for seeing, equals $5-10 \%$.

(iii) Relation to the Granulation: The filigree structures tend to lie between the granules. This is, however, not a strict rule. It seems that in the course of their lifetime the granules move the filigree structures around with velocities of about $1.5 \mathrm{~km} \mathrm{~s}^{-1}$. Some of the crinkles also seem to wash out temporarily until compressed again by a

* On leave from the University of Hawaii, Astronomy Department. 
new granule. The detailed structure of the filigree, therefore, changes significantly over times comparable to the granule lifetime. The overall structure is, however, preserved over much longer periods of time. The granulation pattern when observed in the continuum well outside the $\mathrm{H} \alpha$ line appears very peculiar in that it has substantially decreased in contrast. It appears 'soft' similar to granulation washed-out by seeing. This abnormal granulation can be traced over long times ( $>30 \mathrm{~min}$ ) and coincides in location to the filigree location. It is, therefore, definitely real.

(iv) Relation to the spicules: The filigree structure falls near the center of the $\mathrm{H} \alpha$ chromospheric rosettes. These rosettes consist of dark elongated mottles which should probably be identified with spicules. There is, therefore, at least a coarse relation between the occurrence of spicules and the filigree. There is no clear evidence that variations in the filigree pattern are related to the generation of spicules. Some spicules seem to originate from the spaces between the crinkles. Too few, however, to conclude a definite relation.

(v) Relation to the magnetic field: Beckers studied the filigree with the Universal Birefringent Filter in the magnesium $b_{1}$ and $b_{2}$ lines. It is very well visible in the far wing of the lines (eg. $b_{1} \pm 0.8 \AA$ ). When traced into the line core the structures increase somewhat in size, as they do in $\mathrm{H} \alpha$, and form structures similar to, and perhaps identical with, the so-called photospheric network. In the magnetically sensitive $b_{2}$ line one sees a one-to-one correspondence between these network structures and the magnetic field so that, at least in the layers seen near the core of the $b_{2}$ line, there is a one-to-one correspondence between the filigree structures and the enhancements in the magnetic field. Simon and Zirker (Solar Physics, submitted for publication) using a spectrograph also found that the filigree occurs in regions of enhanced magnetic field. However, in contrast to the filter observations, they found the magnetic field regions to be much more diffuse $\left(2-3^{\prime \prime}\right)$ so that there is not a one-to-one spatial correspondence between filigree and magnetic field structure.

The filigree patterns have the appearance of a scaled-down supergranule network, a suggestion made also by others (e.g. Vrabec). Similar to the structuring of the magnetic fields by the supergranules (causing the magnetic/chromospheric network), there appears to be a structuring by the granulation at a $\sim 20$ times smaller spatial scale and $\sim 100$ times smaller time scale. Beckers' observations suggest a strong compact concentration of the magnetic field on the scale of the filigree whereas Simon and Zirker's observations indicate an enhancement which is less concentrated. The field strength will, of course, depend on the scale of the magnetic structures. It is at least a few hundred gauss. If concentrated on the scale of the filigree its strength may be much higher although it would be hard to see how granules could concentrate fields (and move flux tubes around) with strength as large as a few thousand gauss.

\section{DISCUSSION}

Acton: Do you feel that in the very quiet regions the filigree will be related to the network boundaries. Beckers: In the very quiet regions the magnesium network is easily visible and is concentrated in very 
small elements that are very stable. They have the same appearance as filigree features but we have not proven yet that the two are identical.

Meyer: Is there any chance to see a velocity pattern? It would help enormously for models of these features.

Beckers: The network is much more visible in the blue wing of the magnesium line than in the red wing and $I$ therefore suspect that there is upward motion associated with these features.

Zirin: I am worried about the inference of big fields. There was another paper published recently in which someone measured fields of $5 \mathrm{G}$ and from them inferred fields of thousands of gauss. I really wonder if we should go so far with our estimates of fields. If they are this strong why don't they become sunspots?

Beckers: I share your worries. I am just reporting results that others have obtained. I personally have a hard time accepting fields as large as $3000 \mathrm{G}$; I am willing to consider fields near $1500 \mathrm{G}$.

Deubner: The structure of the filigree very much resembles that of the inverted granular intensity as, for example, measured by Evans and Catalano. In the case of the inverted photospheric intensity you can see in all metal lines bright structures corresponding to the intergranular lanes that are associated with downward flows, so maybe we need to look again at the motion of filigree.

Beckers: There is a tendency for the filigree to occur in the intergranular lanes but the filigree are bright features observed against the dark intergranular lanes. When you observe in the intergranular lanes it will depend entirely on your resolution whether you see something bright or something dark. If your resolution is poor and the filigree contrast is not too great you may still see just the intergranular lanes and the downward movement associated with them. 